CLINICAL STUDY

\title{
Lower early morning plasma cortisol levels are associated with thyroid autoimmunity in the elderly
}

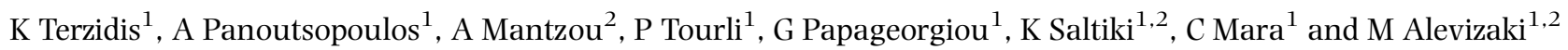 \\ ${ }^{1}$ Endocrine Unit, Department of Medical Therapeutics, Alexandra Hospital and ${ }^{2}$ Endocrine Unit, Evgenideion Hospital, Athens University School of \\ Medicine, 80 Vass. Sofias Avenue, 11528 Athens, Greece
}

(Correspondence should be addressed to M Alevizaki at Athens University School of Medicine; Email: mani@otenet.gr)

\begin{abstract}
Objectives: Thyroid autoimmunity decreases in the very old. We investigated whether glucocorticoid (GC) activity, which increases in old age, is involved in this process.

Subjects and methods: A total of 321 ambulatory subjects (age 51-95 years, median 71, 207 female) were studied. Thyroid function tests, cortisol, glucose, insulin and biochemical parameters were measured. A modified overnight dexamethasone suppression test $(0.25 \mathrm{mg})$ was performed as an index of GC sensitivity.

Results: Forty subjects had positive anti-thyroid peroxidase antibodies and 36 had positive antithyroglobulin antibodies, while 57 had either one or the other or both thyroid autoantibodies (ThAbs) positive. Mean basal cortisol levels were significantly lower in the ThAbs $(+)$ groups $(320 \pm 125$ vs $378 \pm 128 \mathrm{nmol} / \mathrm{l}, P=0.002)$. Triiodothyronine, free thyroxine, post-dexamethasone cortisol levels, C-reactive protein, homeostasis model assessment-insulin-resistance-index and body mass index did not differ between these two groups. Mean age of ThAbs $(+)$ subjects was lower compared to the ThAbs $(-)$ group $(67.38 \pm 7.38$ vs $71.64 \pm 8.57$ years, $P=0.001)$.

Conclusions: Reduced GC activity is associated with an increased prevalence of ThAbs positivity in older ambulatory subjects. Subjects without ThAbs in this population sample are relatively older. It is not known whether this is related to increasing GC activity with age.
\end{abstract}

European Journal of Endocrinology 162 307-313

\section{Introduction}

Immunosenescence is a well-characterized phenomenon accompanying ageing and represents a malfunction of the immune system leading to both increased infection susceptibility and increased frequency of certain kinds of autoimmune disorders in older age $(1,2)$. Several mechanisms have been postulated $(3,4)$ including neuroendocrine changes (5). Of particular interest is the role of the stress and hypothalamuspituitary-adrenal (HPA) axis activity in this process (6). Ageing is accompanied with activation of the HPA axis and increased levels of ACTH and cortisol (7-9). Ageing has also been associated with changes in the immune system sharing many common characteristics with the alterations in immune function seen in chronic stress (10) and/or glucocorticoid (GC) treatment (11).

Autoimmune thyroiditis is a common autoimmune disorder and a typical example of an organ-specific autoimmune disorder. Various studies have assessed the prevalence of thyroid autoimmunity in the normal population. Both female gender and increasing age have been associated with a higher frequency of thyroid antibody positivity $(12,13)$. However, a few studies concerning healthy centenarians and selected groups of ambulatory elderly people have shown decreasing prevalence of thyroid autoimmunity with age, which then approaches that of younger age groups $(14,15)$.

The aim of this study was to assess thyroid autoimmunity in a population sample consisting of apparently healthy elderly subjects and to examine how the ageing process affects this phenomenon; furthermore, possible associations of thyroid autoimmunity with the HPA axis activity and with various metabolic parameters were examined.

\section{Subjects and methods}

We studied a final number of 321 community-dwelling elderly individuals (207 women, 114 men, aged 51-95 years) who were all permanent residents of a rural area in Peloponnese (Southern Greece). All subjects were recruited through an announcement in the local Recreation Centre for the elderly that offered a preventive screening for thyroid function and general health evaluation. Nearly all the elderly ambulatory subjects of this area regularly visit this recreation 
centre, and the majority were female. In total $85 \%$ of the regular attendees presented for evaluation. A detailed medical history (including administered medication) was recorded, and a physical examination was performed (including thyroid gland palpation, arterial blood pressure measurement, weight, height, waist and hip circumference measurements). All the subjects included in this study were born in this area and have lived in this area practically all their lives.

Thyroid function tests (free thyroxine $\left(\mathrm{FT}_{4}\right), \mathrm{TSH}$, triiodothyronine $\left(\mathrm{T}_{3}\right)$, anti-thyroglobulin (TG) and antithyroid peroxidase (TPO) autoantibodies), basal cortisol, glucose, insulin, lipid levels and biochemical profile were determined between 0730 and $0900 \mathrm{~h}$ after an overnight fast. An ultra-low dose $(0.25 \mathrm{mg})$ dexamethasone suppression test (DST) was performed in 232 of the participants in the study, as it was initiated from a certain point onwards, as previously described (16). Subjects ingested $0.25 \mathrm{mg}$ dexamethasone at $2300 \mathrm{~h}$, and a venous blood sample for cortisol, insulin and glucose levels measurement was obtained the following morning (after overnight fast) between 0730 and $0900 \mathrm{~h}$. The level of cortisol suppression, defined as delta-cortisol, was calculated by subtracting postdexamethasone suppression cortisol levels from basal cortisol levels. This test has been used before as an indirect index of hypothalamus-pituitary sensitivity to GC (17).

From the original group who presented for evaluation, subjects with a history of coronary artery disease $(n=31)$, cerebrovascular disease $(n=11)$ and cancer $(n=6)$ were excluded from the study. Furthermore, two individuals who were diagnosed during the study with previously unknown hypothyroidism (TSH levels $>10 \mathrm{mIU} / \mathrm{l})$, three who had abnormal liver function tests and eleven who had creatinine levels above $1.3 \mathrm{mg} / \mathrm{dl}$ were also excluded from the final statistical analysis, leaving a total of 321 subjects that represented the final cohort of the initial analysis. All women were postmenopausal and did not receive hormone replacement therapy. A total of 23 subjects were on $\mathrm{L}_{-} \mathrm{T}_{4}$ treatment and 25 had a past diagnosis of thyroid disease. A significant proportion $(n=63)$ of the studied individuals had diabetes mellitus (defined as either a history of diabetes mellitus, or a fasting glucose level $\geq 7.0 \mathrm{mmol} / \mathrm{l})$. Because cortisol levels may be influenced by the diabetic status and thyroid antibodies presence may be affected by a history of thyroid disease or thyroid function abnormalities, a separate analysis was performed after exclusion of the subjects having a history of diabetes mellitus or a fasting glucose $>6.1 \mathrm{mmol} / \mathrm{l}$ and those with known thyroid disease. The 'apparently normal' group consisted of 224 subjects (14 subjects had both disorders).

The study was approved by the institutional ethics committee, and all subjects gave their informed consent.

Serum insulin, TSH, $\mathrm{FT}_{4}, \mathrm{~T}_{3}$, serum thyroid autoantibodies (ThAbs; anti-TPO and anti-TG) and cortisol were measured using chemiluminescent immunometric assays with the DPC Immulite 2000 (Siemens, Llanberis, Gwynedd, UK). Reference range was TSH 0.36-4 mIU/l, $\mathrm{FT}_{4}$ 9-26 pmol/l, $\mathrm{T}_{3} 1.1-2.9 \mathrm{nmol} / \mathrm{l}$, anti-TPO $<30 \mathrm{IU} / \mathrm{ml}$ and anti-TG $<40 \mathrm{IU} / \mathrm{ml}$. Positive ThAbs were considered if either anti-TPO or anti-TG or both autoantibodies were above the upper normal limit of their normal reference range. The population was then divided into two groups according to their thyroid antibody positivity: ThAbs $(+)$ and ThAbs $(-)$.

The levels of glucose, total cholesterol, high density lipoprotein, low density lipoprotein, triglycerides, C-reactive protein (CRP), alanine aminotransferase (ALT), aspartate aminotransferase (AST), blood urea nitrogen and creatinine were measured using an automated analyzer, Architect c8000, Abbott. Basal insulin-resistance (IR) index (homeostasis model assessment-IR-index) was calculated according to the formula: $\mathrm{IR}=\mathrm{FI} \times \mathrm{G} / 22.5$, where $\mathrm{FI}$, fasting insulin $(\mu \mathrm{IU} / \mathrm{ml})$ and $\mathrm{G}$, fasting glucose $(\mathrm{mmol} / \mathrm{l})$.

\section{Statistical analysis}

Statistical analysis was performed using the SPSS statistical package version 11 (SPSS Inc., Chicago, IL, USA). All descriptive data are presented as mean \pm s.D. Student's $t$-test was used to compare mean values between groups where the distribution was normal, and Mann-Whitney non-parametric test was used when the distribution of values was not normal (i.e. whenever Levene's test for homogeneity of variance was significant). The mean baseline and post-DST cortisol levels were compared using the two-sample Wilcoxon rank-sum test, while the association between them was assessed using a stepwise linear regression model adjusted for age, body mass index (BMI) and liver function tests. The $\chi^{2}$ test was used to analyze the differences in ThAbs frequency in various subgroups of the population. Binary logistic regression analysis was performed to analyze the predictive value of various parameters for the presence of ThAbs (using an adjusted stepwise forward likelihood ratio model). The Hosmer and Lemeshow test was checked for non-significance at each step to ensure that the model adequately fitted the data. The odds ratio (OR) of each variable for the positivity of ThAbs and the respective confidence intervals (CI) were calculated. The significance level ( $P$ value) for all of the performed analyses was defined as $P<0.05$.

\section{Results}

The baseline characteristics of the participating subjects are shown in Table 1. The mean age of the sample population was 70.83 years (range 51-95). The mean age of the male subset $(74.2 \pm 8.2)$ of our population was significantly higher than the mean age of females 
Table 1 Anthropometric and biochemical parameters in the studied population (thyroid autoantibodies (ThAbs) $(+)$, either anti-Tg or anti-TPO or both anti-Tg and anti-TPO positive).

\begin{tabular}{lc}
\hline Male & $n=114(35.5 \%)$ \\
Female & $n=207(64.5 \%)$ \\
Mean age (years) & $70.59( \pm 8.8)$ \\
Body mass index $\left(\mathrm{BMI} ; \mathrm{kg} / \mathrm{m}^{2}\right)$ & $29.6( \pm 5.3)$ \\
Waist-to-hip ratio (W/H) & $0.74( \pm 0.07)$ \\
TSH (mlU/l) & $1.84( \pm 1.87)$ \\
Anti-TPO (+) & $n=40(12.5 \%)$ \\
Anti-TG (+) & $n=36(11.2 \%)$ \\
ThAbs (+) & $n=57(17.8 \%)$ \\
\hline
\end{tabular}

$(69.1 \pm 8.2$ years; $P<0.001)$. The mean BMI of the studied subjects was $29.3 \pm 8.2 \mathrm{~kg} / \mathrm{m}^{2}$. Positive thyroid antibodies (either one or both of them) were found in $17.8 \%$ of the subjects studied. The female subjects had a significantly higher frequency $(23.4 \%)$ of positive ThAbs compared to male subjects $(6.4 \%)$ (Pearson $x^{2}=11615, P=0.001$, Fisher's exact $\left.=0.001\right)$.

Mean TSH was $1.84 \mathrm{mIU} / \mathrm{l}$ and did not differ between male and female subjects (Table 1). The prevalence of subclinical hypothyroidism (defined as TSH 4.5$10 \mathrm{mIU} / \mathrm{l}$ ) in our sample population was $3.2 \%$. The mean age of subjects with subclinical hypothyroidism $(72.56 \pm 9.6)$ did not differ from subjects with TSH $<4.5 \mathrm{mIU} / \mathrm{l}(70.65 \pm 8.5$ years; $P=0.51)$. Mean age and TSH levels did not differ significantly between those with and without goitre. The mean age in subjects with goitre was $71.35 \pm 8.7$ and in subjects without goitre was $70.95 \pm 8.3(P=0.76)$, and the mean TSH in subjects with goitre was $1.73 \pm 0.97$ and in subjects without goitre was $1.81 \pm 0.89, P=0.53$.

The differences of various parameters studied in this population according to their thyroid antibody positivity status are summarized in Table 2 . The mean age of the ThAbs $(+)$ group $(67.38 \pm 7.38$ years $)$ was significantly lower than the mean age of the ThAbs (-)

Table 2 Comparison of various parameters according to thyroid autoantibody positivity (mean \pm S.D).

\begin{tabular}{lccl}
\hline & ThAbs $(-)$ & ThAbs $(+)$ & \multicolumn{1}{c}{$\boldsymbol{P}$} \\
\hline Age (years) & $71.64( \pm 8.57)$ & $67.38( \pm 7.38)$ & $0.001^{\mathrm{a}}$ \\
$\mathrm{TSH}(\mathrm{mlU} / \mathrm{l})$ & $1.61( \pm 0.98)$ & $2.36( \pm 1.83)$ & $0.02^{\mathrm{b}}$ \\
$\mathrm{T}_{3}(\mathrm{nmol} / \mathrm{l})$ & $1.2( \pm 0.24)$ & $1.18( \pm 0.24)$ & $\mathrm{NS}$ \\
$\mathrm{FT}_{4}(\mathrm{pmol} / \mathrm{l})$ & $16.9( \pm 3.1)$ & $16.2( \pm 3.9)$ & $\mathrm{NS}$ \\
Baseline cortisol & $378( \pm 128)$ & $320( \pm 125)$ & $0.002^{\mathrm{a}}$ \\
$\quad(\mathrm{nmol} / \mathrm{l})$ & & & \\
Cortisol post & $168( \pm 118)$ & $143( \pm 90)$ & $\mathrm{NS}$ \\
$\quad$ 0.25 mg DST & & & \\
$\quad(\mathrm{nmol} / \mathrm{l})$ & & & \\
CRP (IU/l) & $0.33( \pm 0.5)$ & $0.24( \pm 0.25)$ & $\mathrm{NS}$ \\
Waist-to-hip ratio & $0.92( \pm 0.07)$ & $0.91( \pm 0.069)$ & $\mathrm{NS}$ \\
HOMA index & $2.2( \pm 1.93)$ & $2.6( \pm 3.75)$ & $\mathrm{NS}$ \\
Body mass index & $29.4( \pm 5.2)$ & $30.5( \pm 5.9)$ & $\mathrm{NS}$ \\
$\quad$ (BMI; kg/m $\left.{ }^{2}\right)$ & & & \\
\hline
\end{tabular}

aStudent's test

${ }^{\mathrm{b}}$ Mann-Whitney test. group (71.64 \pm 8.57 years). Baseline cortisol and TSH levels also differed significantly: the ThAbs $(+)$ group had significantly lower mean cortisol and significantly higher mean TSH levels compared to the ThAbs $(-)$ group (see Table 2). These results were similar when only the 'apparently normal' subjects were included. In the 'apparently normal' ThAbs (+) group, the mean age was $66.94 \pm 7.2$ years (compared to $71.85 \pm 8.4$ years in the ThAbs ( - ) group, $P=0.02$, Student's test), mean cortisol levels were $313.78 \pm 105.6 \mathrm{nmol} / \mathrm{l}$ (compared to $376.81 \pm 123.2 \mathrm{nmol} / \mathrm{l}$ in the ThAbs ( - ) group, $P=0.06$, Student's test) and TSH levels were $2.2 \pm 1.2 \mathrm{mUI} / \mathrm{l}$ (compared to $1.67 \pm 0.82 \mathrm{mUI} / \mathrm{l}$ in the ThAbs $(-)$ group, $P=0.05$, Mann-Whitney test). The results were also not different when the same analysis was performed with each of the ThAbs (anti-TPO and anti-TG) used individually as the grouping variable (data not shown). Mean cortisol levels of males $(390 \pm 129 \mathrm{nmol} / \mathrm{l})$ were significantly higher than mean cortisol levels of females $(353 \pm 126 \mathrm{nmol} / \mathrm{l} ; P=0.016)$.

The mean levels of post-DST cortisol (164.2 $\pm 113.5 \mathrm{nmol} / \mathrm{l})$ compared to the mean baseline cortisol levels $(367.3 \pm 129.3 \mathrm{nmol} / \mathrm{l})$ were significantly lower $(P<0.001$, two-sample Wilcoxon rank-sum test). The association of baseline cortisol with post-DST cortisol levels was studied using a stepwise multiple linear regression model adjusted for age, BMI and liver function tests (ALT and AST aminotransferases). Only baseline cortisol levels $(r=0.322, P<0.001)$ and age $(r=0.158, P=0.014)$ had a significant positive correlation with post-DST cortisol levels. The degree of cortisol suppression (delta-cortisol) did not differ according to thyroid antibodies presence (189.7 \pm 130.7 in the ThAbs $(-)$ group and $177.4 \pm 149.9$ in the ThAbs $(+)$ group, $P=0.580$.) Dexamethasone concentrations were not measured in this study.

The sample population was divided arbitrarily into three age intervals (each including a 15-year-range): '50-65 years', '65-80 years' and '>80 years'. The prevalence of thyroid autoantibody positivity was calculated in each of these groups, and the resulting frequencies are illustrated in Fig. 1. Totally, 34\% of individuals aged 50-65 years had positive ThAbs, whereas the ThAbs frequency for the age groups '65-80 years' and '>80 years' was 18 and $4 \%$ respectively (Fig. 1). These differences remained significant when the age groups were analyzed in pairs. The ' $50-65$ years' group compared to the ' $65-80$ years' group had a significantly higher rate of ThAbs positivity (Pearson $x^{2}=6.73, P=0.009$, Fisher's exact $=0.012$ ). The ' $65-80$ years' group compared to the ' $>80$ years' group also had a significantly higher frequency of positive ThAbs (Pearson $x^{2}=6.53, P=0.011$, Fisher's exact $=0.012$ ).

The value of age and cortisol as predictors for the positivity of ThAbs was studied using logistic regression analysis. A stepwise forward likelihood ratio model 


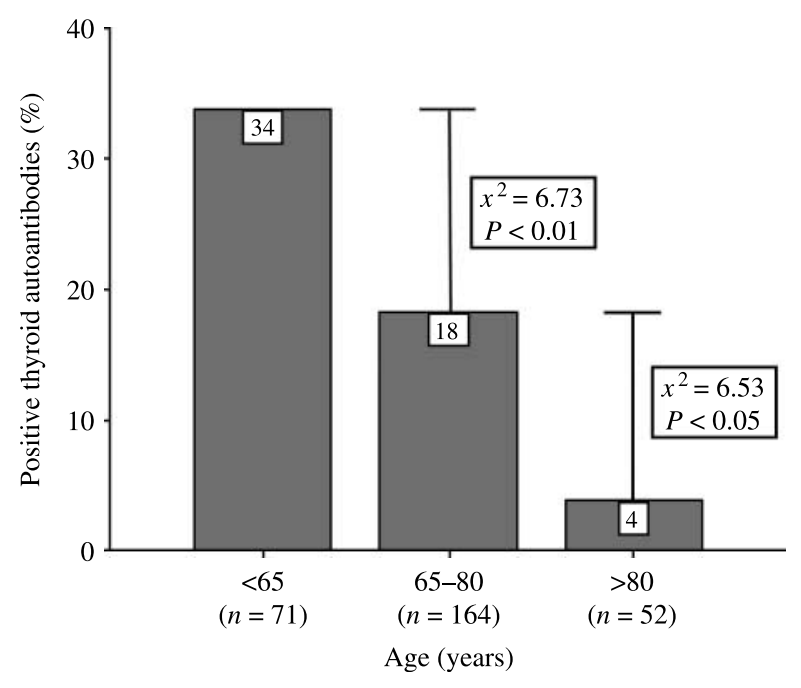

Figure 1 Prevalence of thyroid autoantibodies in the three different age groups of elderly ambulatory individuals; statistical difference in comparison with preceding group.

adjusted for gender, body weight, serum creatinine, glucose, the presence of goitre and CRP levels was used. The adjusted OR for positive ThAbs for 1-year increase in age was 0.957 (95\% CI: 0.921-0.995), and the respective adjusted OR for $10 \mathrm{nmol} / \mathrm{l}$ increase in cortisol levels was 0.969 (95\% CI: 0.943-0.995). The predictive value of both age and cortisol remained significant when the same analysis was conducted in the 'apparently normal' group. Similar results were obtained when subjects with TSH $<0.4$ were excluded (Table 3).

When 'apparently normal' female subjects were studied separately, both age and basal cortisol remained significant predictors for ThAbs positivity $(\mathrm{OR}=0.937$, 95\% CI: $0.892-0.984$ and $\mathrm{OR}=0.967,95 \% \mathrm{CI}$ : 0.935-0.999 respectively). On the other hand, when

Table 3 Effect of age and baseline morning cortisol levels on the presence of positive thyroid autoantibodies (ThAbs; stepwise forward likelihood ratio model adjusted for gender, the presence of goitre, body weight, serum creatinine, glucose and C-reactive protein levels) in elderly ambulatory subjects.

\begin{tabular}{lcc}
\hline & $\begin{array}{c}\text { Odds ratio for } \\
\text { ThAbs positivity }\end{array}$ & CI (95\%) \\
\hline All subjects & 0.957 & $0.921-0.995$ \\
Age $^{\mathrm{a}}$ & 0.969 & $0.943-0.995$ \\
Cortisol $^{\mathrm{b}}$ & & \\
\multicolumn{3}{c}{ Subjects with no diabetes or known thyroid dysfunction and with $^{\text {TSH levels 0.4-3.6 mU/l }}$} \\
Age $^{\mathrm{a}}$ & 0.932 & $0.888-0.978$ \\
Cortisol $^{\mathrm{b}}$ & 0.951 & $0.916-0.988$ \\
\hline
\end{tabular}

$\mathrm{Cl}$, confidence intervals

${ }^{a}$ One year increase in age.

b $10 \mathrm{nmol} / \mathrm{l}$ increase in baseline cortisol levels. only the male subjects were studied, then cortisol levels remained a significant predictor variable (OR: 0.810, 95\% CI: 0.695-0.943), while age did not ( $\mathrm{OR}=1.092$, $95 \% \mathrm{CI}=0.910-1.311, P=0.34)$.

\section{Discussion}

Results from studies examining thyroid autoimmunity prevalence are not directly comparable due to the diversity in the applied biochemical methodology, the study design and different population characteristics. The overall prevalence of positive ThAbs found in this cross-sectional study $(17.8 \%)$ is similar to that reported in other epidemiological studies $(12,13)$. The female predominance in thyroid autoimmunity, which has been found in many other studies, was also confirmed. Furthermore, we found a decrease in the prevalence of ThAbs associated with age in females. At first sight, this finding appears to be in contrast to other studies where an age-associated increase in thyroid autoimmunity has been found in females $(12,13)$. However, in those studies, women of all age groups were included, whereas in the current study the participating subjects were all above 50 years (the oldest was 95 years old, the median age was 71 years); the population studied by Pedersen et al. (12) had an age range from 18 to 65 years, while the study by Hollowell et al. (NHANES III) (13) covered practically all age groups. A decreased prevalence of thyroid autoimmunity in older age has been reported before in studies examining healthy centenarians and a selected population of healthy elderly people aged more than 65 years $(14,15)$; our results concur with the latter studies.

It has been hypothesized that the decline in thyroid autoimmunity observed in the older subgroup of elderly females may be a consequence of a selection effect (15). As the presence of positive ThAbs has been associated with increased cardiovascular risk $(18,19)$, one could speculate that patients with Hashimoto's thyroiditis may have a higher mortality rate at a younger age than subjects with negative ThAbs (15).

It should be pointed out that our population consisted of apparently healthy elderly subjects. Subjects with serious co-morbidities were excluded from our final analysis. It is thus possible that the age-associated decline in ThAbs is specifically found in studies including only disease-free females aged above 65 years. In studies performed in unselected populations (including hospitalized patients), the rise in thyroid antibody frequency with age may persist in the older age groups (2).

It should be noted that in the NHANES III study that also included elderly ambulatory persons (13), a steady rise in thyroid antibodies prevalence was noticed even in the 'above 80 years female disease-free' group (reaching $26.5 \%$ frequency of positive anti-TPO antibodies). Differences in iodine supply may contribute to this 
difference (12), and while iodine adequacy has not been evaluated in our study, previous studies from south-western Greece, where this study was conducted, showed that this is an iodine-replete area $(20,21)$. Another possible explanation is differences in the prevalence of subclinical hypothyroidism. The overall frequency of thyroid dysfunction (defined as TSH $>4.5 \mathrm{mIU} / \mathrm{l}$ ) in our sample was $3.8 \%$ (including those with subclinical hypothyroidism and two subjects with clinical hypothyroidism), whereas in the NHANES III trial it was $4.7 \%$. In our study, an age-associated increase in subclinical hypothyroidism was not demonstrated, contrary to the NHANES III study results. One further factor that might be influencing the presence of ThAbs could be the presence of longstanding goitre (22). However, in our cohort, the presence of goitre did not have any influence, possibly because the age range studied was rather narrow.

One interesting mechanism that might be involved in the lower prevalence of thyroid autoimmunity in the very old could be alterations in the HPA axis that we explored in our study. We mainly used baseline early morning cortisol levels for HPA axis activity assessment. Basal morning cortisol may not always be representative of the 24-h cortisol production; however, it has been previously shown to have low intraindividual variability and to correlate well with the feedback sensitivity of the HPA axis (an association confirmed in our study) (16). Cortisol levels were lower in those with positive ThAbs, and this association was independent of age. Further analysis, according to gender, showed that this association was significant in both the male and female 'apparently normal' subsets of our population. Decreased GC activity has previously been associated with thyroid autoimmunity both in humans (23-26) and in laboratory models of autoimmune thyroid disease (27). As to the mechanisms that have been suggested to be involved in such associations, it is worth mentioning that hypercortisolaemia (due to stress or ageing) may lead to decreased hypothalamus and pituitary sensitivity to GCs, subsequent activation of the HPA axis and increased peripheral GC action ultimately leading to significant immune function changes, mainly consisting of a reduced pool of naive T-cells and a cytokine profile shift from a Th1 to a Th2 response (6). The Th2 type immune response as a result of GC action seems to have a protective role against certain types of autoimmunity (26). However, it should be noted that in our study, we simply found cortisol levels in the ThAbs positive group that were lower than the relatively 'normal' levels of the ThAbs negative group. Therefore, a protective role of increased GC activity on thyroid autoimmunity cannot be assumed based on the results of our study.

One further factor that might be involved in this process could be the effect of age on cortisol levels. A lot of studies have examined the alterations of cortisol metabolism occurring through ageing. We have found that baseline cortisol levels increase with age (28), a finding compatible with other studies $(7,9)$. It is not known if this age-associated increase in cortisol levels contributes to the declining thyroid autoimmunity in the elderly. In our study, both cortisol levels and age were found to be independent determinants of decreased prevalence of thyroid autoimmunity but the extent of this interaction is difficult to identify.

We also examined the possibility that sensitivity to GCs might play a role as GC negative feedback inhibition also seems to be impaired in the elderly $(29,30)$. In our study, we used the $0.25 \mathrm{mg}$ DST as a means of assessing central feedback sensitivity to GCs $(16,17)$. Using this indirect index of GC sensitivity, we confirmed the decreased sensitivity of the HPA axis in the elderly, but we did not find any association with ThAbs presence. Our study may not have been powerful enough to reveal such an association. It should be noted, however, that the ultra-low dose DST is only a rough index of sensitivity to GCs, only suitable for population studies. Therefore, it is not clear whether the observations that we made have the same significance for ThAbs positivity. Another possible explanation that should be discussed is that patients with positive ThAbs may also have more frequently some form of subclinical autoimmune adrenalitis, which may account for the lower observed baseline cortisol levels. ACTH levels were not measured to assess this possibility; however, one has to note that the presence of coexisting autoimmune diseases is rather rare in patients with thyroid autoimmunity (31).

The present study has some obvious limitations, including its cross-sectional nature. The population sample studied might not be representative of the general population since it included only ambulatory elderly people and only the subjects who responded to the invitation for a health survey. It should be noted, however, that over $85 \%$ of the population participated, and that our results concerning the prevalence of autoimmune thyroiditis are consistent (even though not directly comparable) with other epidemiological studies and that the conclusions are roughly similar. The subjects examined in our study were overweight, although this did not appear to influence the association of cortisol and age with thyroid antibody positivity. It is also possible that an age-associated decline in thyroid autoimmunity in men may have not been detected due to the small number of male participants and the low prevalence of thyroid autoimmunity in males.

In conclusion, our study confirmed that the prevalence of thyroid autoimmunity decreases in the apparently healthy elderly. It further confirmed that the occurrence of thyroid autoimmunity has significant differences between males and females. The decreasing prevalence of thyroid autoimmunity in the healthy elderly appeared to be female specific. The results of our study taken together with other epidemiological 
studies show a gradual rise of thyroid autoimmunity prevalence in females until approximately a decade after menopause and thereafter a gradual decline. One cannot exclude the possibility that the ageassociated increase in cortisol levels may contribute to the declining thyroid autoimmunity in the elderly as we found that both cortisol levels and age were independent determinants of decreased prevalence of thyroid autoimmunity; however, the extent of this interaction cannot at present be clarified.

\section{Declaration of interest}

The authors declare that there is no conflict of interest that could be perceived as prejudicing the impartiality of the research reported.

\section{Funding}

This research did not receive any specific grant from any funding agency in the public, commercial, or not-for-profit sector.

\section{Acknowledgements}

We should like to acknowledge the municipality of Lygourio who helped in the recruitment of individuals for this study.

\section{References}

1 Ostan R, Bucci L, Capri M, Salvioli S, Scurti M, Pini E, Monti D \& Franceschi C. Immunosenescence and immunogenetics of human longevity. Neuroimmunomodulation 200815 224-240.

2 Pinchera A, Mariotti S, Barbesino G, Bechi R, Sansoni P, Fagiolo U, Cossarizza A \& Franceschi C. Thyroid autoimmunity and ageing. Hormone Research $1995 \mathbf{4 3}$ 64-68.

3 Yung RL \& Julius A. Epigenetics, aging, and autoimmunity. Autoimmunity $2008 \mathbf{4 1}$ 329-335.

4 Krichevsky S, Pawelec G, Gural A, Effros RB, Globerson A, Yehuda DB \& Yehuda AB. Age related microsatellite instability in T cells from healthy individuals. Experimental Gerontology 200439 507-515.

5 Arlt $\mathrm{W} \&$ Hewison M. Hormones and immune function: implications of aging. Aging Cell 20043 209-216.

6 Bauer ME. Stress, glucocorticoids and ageing of the immune system. Stress 20058 69-83.

7 Purnell JQ, Brandon DD, Isabelle LM, Loriaux DL \& Samuels MH. Association of 24-hour cortisol production rates, cortisol-binding globulin, and plasma-free cortisol levels with body composition, leptin levels, and aging in adult men and women. Journal of Clinical Endocrinology and Metabolism 200489 281-287.

8 Ferrari E, Cravello L, Muzzoni B, Casarotti D, Paltro M, Solerte SB, Fioravanti M, Cuzzoni G, Pontiggia B \& Magri F. Age-related changes of the hypothalamic-pituitary-adrenal axis: pathophysiological correlates. European Journal of Endocrinology 2001144 319-329.

9 Van Cauter E, Leproult R \& Kupfer DJ. Effects of gender and age on the levels and circadian rhythmicity of plasma cortisol. Journal of Clinical Endocrinology and Metabolism 199681 2468-2473.

10 McEwen BS, Biron CA, Brunson KW, Bulloch K, Chambers WH, Dhabhar FS, Goldfarb RH, Kitson RP, Miller AH, Spencer RL \& Weiss JM. The role of adrenocorticoids as modulators of immune function in health and disease: neural, endocrine and immune interactions. Brain Research. Brain Research Reviews 199723 79-133.
11 Bauer ME, Papadopoulos A, Poon L, Perks P, Lightman SL, Checkley S \& Shanks N. Dexamethasone-induced effects on lymphocyte distribution and expression of adhesion molecules in treatment-resistant depression. Psychiatry Research 2002 $1131-15$.

12 Pedersen IB, Knudsen N, Jorgensen T, Perrild H, Ovesen L \& Laurberg P. Thyroid peroxidase and thyroglobulin autoantibodies in a large survey of populations with mild and moderate iodine deficiency. Clinical Endocrinology 200358 36-42.

13 Hollowell JG, Staehling NW, Flanders WD, Hannon WH, Gunter EW, Spencer CA \& Braverman LE. Serum TSH T(4), and thyroid antibodies in the United States population (1988 to 1994): National Health and Nutrition Examination Survey (NHANES III). Journal of Clinical Endocrinology and Metabolism 200287 489-499.

14 Mariotti S, Barbesino G, Caturegli P, Bartalena L, Sansoni P, Fagnoni F, Monti D, Fagiolo U, Franceschi C \& Pinchera A. Complex alteration of thyroid function in healthy centenarians. Journal of Clinical Endocrinology and Metabolism $1993 \mathbf{7 7}$ 1130-1134.

15 Mariotti S, Sansoni P, Barbesino G, Caturegli P, Monti D, Cossarizza A, Giacomelli T, Passeri G, Fagiolo U \& Pinchera A. Thyroid and other organ-specific autoantibodies in healthy centenarians. Lancet 1992339 1506-1508.

16 Huizenga NA, Koper JW, de Lange P, Pols HA, Stolk RP, Grobbee DE, de Jong FH \& Lamberts SW. Interperson variability but intraperson stability of baseline plasma cortisol concentrations, and its relation to feedback sensitivity of the hypothalamo-pituitary-adrenal axis to a low dose of dexamethasone in elderly individuals. Journal of Clinical Endocrinology and Metabolism $19988347-54$.

17 van Rossum EF, Koper JW, van den Beld AW, Uitterlinden AG, Arp P, Ester W, Janssen JA, Brinkmann AO, de Jong FH, Grobbee DE, Pols HA \& Lamberts SW. Identification of the BclI polymorphism in the glucocorticoid receptor gene: association with sensitivity to glucocorticoids in vivo and body mass index. Clinical Endocrinology 200359 585-592.

18 Stamatelopoulos KS, Kyrkou K, Chrysochoou E, Karga H, Chatzidou S, Georgiopoulos G, Georgiou S, Xiromeritis K, Papamichael $\mathrm{CM} \&$ Alevizaki M. Arterial stiffness but not intima-media thickness is increased in euthyroid patients with Hashimoto's thyroiditis: the effect of menopausal status. Thyroid $200919857-862$.

19 Xiang GD, He YS, Zhao LS, Hou J, Yue L \& Xiang HJ. Impairment of endothelium-dependent arterial dilation in Hashimoto's thyroiditis patients with euthyroidism. Clinical Endocrinology $200664698-702$.

20 Michalaki M, Kyriazopoulou V, Paraskevopoulou P, Vagenakis AG \& Markou KB. The odyssey of nontoxic nodular goiter (NTNG) in Greece under suppression therapy, and after improvement of iodine deficiency. Thyroid $2008 \mathbf{1 8} 641-645$.

21 Koutras DA, Alevizaki M, Tsatsoulis A \& Vagenakis AG. Greece is iodine sufficient. Lancet $2003 \mathbf{3 6 2} 405-406$.

22 Aghini-Lombardi F, Antonangeli L, Martino E, Vitti P, Maccherini D, Leoli F, Rago T, Grasso L, Valeriano R, Balestrieri A \& Pinchera A. The spectrum of thyroid disorders in an iodine-deficient community: the Pescopagano survey. Journal of Clinical Endocrinology and Metabolism 199984 561-566.

23 Kokandi AA, Parkes AB, Premawardhana LD, John R \& Lazarus JH. Association of postpartum thyroid dysfunction with antepartum hormonal and immunological changes. Journal of Clinical Endocrinology and Metabolism 200388 1126-1132.

24 Colao A, Pivonello R, Faggiano A, Filippella M, Ferone D, Di SC, Cerbone G, Marzullo P, Fenzi G \& Lombardi G. Increased prevalence of thyroid autoimmunity in patients successfully treated for Cushing's disease. Clinical Endocrinology 200053 13-19.

25 Takasu N, Komiya I, Nagasawa Y, Asawa T \& Yamada T. Exacerbation of autoimmune thyroid dysfunction after unilateral adrenalectomy in patients with Cushing's syndrome due to an adrenocortical adenoma. New England Journal of Medicine 1990 322 1708-1712. 
26 Sternberg EM. Neuroendocrine regulation of autoimmune/ inflammatory disease. Journal of Endocrinology $2001 \quad 169$ 429-435.

27 Wick G, Hu Y, Schwarz S \& Kroemer G. Immunoendocrine communication via the hypothalamo-pituitary-adrenal axis in autoimmune diseases. Endocrine Reviews $199314539-563$.

28 Alevizaki M, Tourli P, Terzidis K, Papageorgiou G, Saltiki K \& Mantzou E. Morning plasma cortisol levels and metabolic parameters in elderly ambulatory subjects. 90th Annual Meeting of the Endocrine Society, ENDO 2008, San Fransisco, Book of Abstracts, 2008 426-427 (abstract).

29 Wilkinson CW, Petrie EC, Murray SR, Colasurdo EA, Raskind MA \& Peskind ER. Human glucocorticoid feedback inhibition is reduced in older individuals: evening study. Journal of Clinical Endocrinology and Metabolism 200186 545-550.

30 Wilkinson CW, Peskind ER \& Raskind MA. Decreased hypothalamic-pituitary-adrenal axis sensitivity to cortisol feedback inhibition in human aging. Neuroendocrinology 1997 65 79-90.

31 Jenkins RC \& Weetman AP. Disease associations with autoimmune thyroid disease. Thyroid 200212 977-988.

Received 2 November 2009

Accepted 4 November 2009 